Relations industrielles

Industrial Relations

\title{
Psychology in Management, by Mason HAIRE, McGraw-Hill Book Company, 220 pp., 1956.
}

\section{C.-R. Giroux}

Volume 11, numéro 3, juin 1956

URI : https://id.erudit.org/iderudit/1022642ar

DOI : https://doi.org/10.7202/1022642ar

Aller au sommaire du numéro

Éditeur(s)

Département des relations industrielles de l’Université Laval

ISSN

0034-379X (imprimé)

1703-8138 (numérique)

Découvrir la revue

Citer ce compte rendu

Giroux, C.-R. (1956). Compte rendu de [Psychology in Management, by Mason HAIRE, McGraw-Hill Book Company, 220 pp., 1956.] Relations industrielles / Industrial Relations, 11(3), 225-226. https://doi.org/10.7202/1022642ar

Tous droits réservés @ Département des relations industrielles de l’Université Laval, 1956
Ce document est protégé par la loi sur le droit d'auteur. L’utilisation des services d'Érudit (y compris la reproduction) est assujettie à sa politique d'utilisation que vous pouvez consulter en ligne.

https://apropos.erudit.org/fr/usagers/politique-dutilisation/ 
gue de métier, mais bien à l'étudiant et à l'administrateur. Son livre en est un, moins de création que d'agencement ordonné et logique. Il puise abondamment au passé de la sociologie industrielle; les sources sont nombreuses, et couvrent généreusement jusqu’à l'année 1955, ce qui constitue décidément un mérite et un avantage.

L'auteur s'intéresse, après bien d'autres, aux relations humaines dans l'industrie. A l'aide d'un schème et de concepts sociologiques - tels interaction, images mentales, situation, rôle, statuts, groupe, culture, changement - il réussit à bâtir une image cohérente de la réalité industrielle.

Il passe plus de la moitié du volume à décrire les relations humaines dans l'entreprise industrielle, sans apporter au sujet beaucoup d'éléments nouveaux. Mais son mérite le plus grand réside dans sa façon, assez généreuse, de traiter la relation entreprise-localité. Ici, il analyse la nature changeante des agglomérations urbaines et des interrelations qui s'y nouent; il s'attaque ensuite au problème de psychologie collective qu'est l'insécurité latente des masses ouvrières; il étudie plus loin la mobilité sociale dans la localité industrielle, les problèmes de relations ethniques et raciales qu'elle pose, ainsi que les relations qu'elle établit avec la direction des entreprises et le syndicalisme ouvrier. Cette section, systématique à souhait, fait quelque peu oeuvre de pionnier, et, peu prétentieuse, mérite une lecture attentive de la part de l'étudiant à la recherche d'une bonne introduction.

Une dernière section, qui marche davantage dans les sentiers battus, appose industrie et société, décrivant l'image d'une société en voie d'industrialisation, y étudiant le rôle de la direction des entreprises et du syndicalisme, faisant intervenir le gouvernement, et, finalement, posant le dilemne: équilibre ou «anomie ».

Une oeuvre qui dépasse le niveau de la simple vulgarisation, et qui ouvre d'utiles horizons à l'étudiant comme au «praticien».

Psychology in Management, by Mason Haire, McGraw-Hill Book Company, 220 pp., 1956.

Ce volume de sept chapitres touche à presque tous les problèmes humains qui peuvent surgir dans l'administration de l'entreprise, ceci depuis la sélection des employés jusqu’à la structure interne de l'entreprise. Cependant, il n'y est pas question de théories ni de formules; ?approche est purement psychologique sans rendre difficile la compréhension du texte.

Les administrateurs et les gérants de sociétés commerciales et industrielles seront très intéressés par le thème domirant de ce livre: Comment pourra-t-on maintenir et augmenter l'intérêt de l'employé dans son travail, alors qu'une semaine de travail de plus en plus courte lui laisse plus de temps à consacrer à des loisirs qui accaparent son esprit même au travail ?

L'auteur s'est servi d'une loi fondamentale des théories de l'apprentissage pour expliquer les mécanismes de développements de ces problèmes humains. Cette loi, la loi de l'effet, peut se lire comme suit: Une activité est fixée ou éliminée selon qu'elle a pour effet de satisfaire ou non la motivation de l'individu. N'oublions pas qu'il est naturel à tout organisme vivant de répéter les actions qui lui procurent une certaine satisfaction.

«Behavior which seems to be rewarded tends to be repeated, while that which seems not to lead to reward or seems to lead to punishment tends to be eliminated. »

$\mathrm{Si}$ les patrons approfondissaient et appliquaient cette loi, ils auraient selon Haire une belle opportunité pour modifier le comportement de leurs employés. Nul doute que l'auteur aurait pu illustrer sa pensée avec les lois d'exercice, de fréquence, d'intensité, etc., mais leur usage aurait rendu la lecture du texte beaucoup plus difficile.

Parlant de l'intérêt grandissant dans les relations humaines, Haire expose les circonstancés qui ont forcé les administrateurs et les employeurs à s'en occuper:

1-L'oubli volontaire dans le passé des problèmes humains de l'entreprise, à cause de la résistance de l'homme à tout changement.

2-L'évolution rapide de la culture et de l'économie depuis cinquante ans.

3-L'augmentation de la sécurité du travailleur par suite des contrats de travail.

4-L'application croissante des principes de l'automation.

Ces facteurs seraient à l'origine de l'insouciance et du manque d'initiative manifestés par les employés. La sécurité de l'emploi est telle que l'employé a appris (la loi de l'effet) que, dans la 
majorité des cas, la durée de l'emploi est la seule condition pour obtenir une promotion. Les employeurs sont à Elâmer pour cet état de choses, puisqu'ils n'ont pas su fournir des récompenses distinctives pour la durée de l'emploi et pour l'intérêt et l'initiative.

Dans son chapitre sur la motivation humaine, Haire se base sur la hiérarchie des besoins pour expliquer la motivation humaine. Selon cette théorie, l'intensité des besoins détermine leur importance relative. Il a été impossible à date de vérifier la validité de cet énoncé au niveau humain, de sorte que cette notion d'hiérarchie est remplacée progressivement par la notion d'adaptation, qui décrit mieux la flexibilité du comportement humain. En effet, cette notion semble plus proche de la réalité. Comme les besoins naissant en séries, sont presque illimités et que par contre la possibilité de les satisfaire est plutôt limitée, il est concevable que l'industriel le mieux équilibré attribue plus d'importance aux besoins les plus compatibles avec le milieu dans lequel il vit, car il peut alors les satisfaire plus facilement. En d'autres mots, il adapte ses besoins aux circonstances.

Cet exposé sur la nature humaine est secondaire si l'on considère que l'auteur cherche surtout à faire saisir l'influence des besoins sur la perception et le comportement humain. Tout au long de ses trois meilleurs chapitres - - Leadership and Supervision, Communication anl Training》 - appuie constamment sur le fait que le comportement de l'employé est déterminé par sa perception, puisqu'elle structure son champ d'activité, son environnement. Haire explique phusieurs fois que les employeurs se trompent quand ils se basent sur leur propre compréhension d'une situation pour chercher à changer le comportement et les attitudes de leurs employés. L'attaque directe et logique s'est avérée inefficace, alors qu'en modifiant la perception des employés, les employeurs peuvent espérer des résultats concrets.

Dans un excellent chapitre sur les communications, Haire fait une étude psychologique très poussée sur les phénomènes perceptuels de distorsion, d'omission et de rejet inhérents aux communications. Il présente quatre principes fondamentaux à de saines communications:

1 «...tailor information on the basis of the recipient's organization »;

2 -.. transmit information in small units $\gg$;

3 - «...p provide an opportunity for «feedback» from the recipient »;

4 «... provide an opportunity for participation on the part of the recipient. 》

Ce volume qui s'attaque à des problèmes dynamiques et pressants mérite d'être lu et relu par tous et spécialement par tous ceux qui ont affaire à des subalternes. Haire ne solutionne pas les problèmes qu'il décrit, puisque leur solution dépend de la situation dans laquelle ils se déroulent. Toutefois, il offre des principes qui peuvent être utilisés à cette fin. Il est regrettable que chaque chapitre ne soit pas suivi d'une bonne bibliographie et de références.

«If we see the superior's job as one of acomplishing production through people, then it is the people, not the production, that must be his first consideration. 》

C.-R. Giroux 